\title{
ASYMPTOTIC BEHAVIOR OF SOLUTIONS OF SECOND ORDER DIFFERENTIAL EQUATIONS WITH INTEGRABLE COEFFICIENTS
}

\author{
BY
}

MANABU NAITO

\begin{abstract}
The differential equation $x^{\prime \prime}+a(t) f(x)=0, t>0$. is considered under the condition that $\lim _{t \rightarrow x} \int^{t} a(s) d s$ exists and is finite, and necessary and/or sufficient conditions are given for this equation to have solutions which behave asymptotically like nontrivial linear functions $c_{1}+c_{2} t$.
\end{abstract}

1. Introduction. We study the asymptotic behavior of solutions of the second order differential equation

$$
x^{\prime \prime}+a(t) f(x)=0, \quad t>0,
$$

under the following assumptions: (i) $a(t)$ is continuous on $[0, \infty)$; (ii) $f(x)$ has a continuous derivative for $x \neq 0$ and satisfies $x f(x)>0, f^{\prime}(x) \geqslant 0$ for $x \neq 0$. A typical example of (1.1) is the generalized Emden-Fowler equation

$$
x^{\prime \prime}+a(t)|x|^{\gamma} \operatorname{sgn} x=0, \quad t>0,
$$

where $\gamma$ is a positive constant. The aim of this paper is to obtain sufficient and/or necessary conditions for (1.1) to have solutions which behave like nontrivial linear functions $c_{1}+c_{2} t$ as $t \rightarrow \infty$. It is known that if $\lim _{t \rightarrow \infty} \int{ }^{t} a(s) d s=\infty$, then all continuable solutions of (1.1) are oscillatory (Bhatia [1], Wong [15]), so, in this case, (1.1) never has a solution which behaves like $c_{1}+c_{2} t$ as $t \rightarrow \infty$. Our particular interest, therefore, is to consider the case in which $a(t)$ satisfies the condition that

$$
\lim _{t \rightarrow \infty} \int^{t} a(s) d s \text { exists and is finite. }
$$

If (1.3) is satisfied, one may introduce the function $A(t)$ defined by

$$
A(t)=\int_{t}^{\infty} a(s) d s, \quad t \geqslant 0 .
$$

Throughout the paper, (1.3) is tacitly assumed, and $A(t)$ always denotes the function defined by (1.4). Note that the integral of (1.4) may converge conditionally.

Under condition (1.3) various nonoscillation criteria have been given by numerous authors; see e.g. Willett [13], Wong [16] (the linear case) and Butler [2], Kwong and Wong [8] (the nonlinear case). In this paper it is shown that for a wide class of equations, not only the existence of a nonoscillatory solution can be determined, but also an explicit asymptotic form of the nonoscillatory solution can be obtained.

Received by the editors December 13, 1982.

1980 Mathematics Subject Classification. Primary 34C11; Secondary 34C 10, 34C 15.

Key words and phrases. Asymptotic integration, asymptotic behavior, differential equations. 
Moreover, if $A(t)$ does not change signs, then we can establish necessary and sufficient conditions for (1.1) to have solutions with specified asymptotic behavior as $t \rightarrow \infty$. In $\$ 2$ we deal with solutions which behave asymptotically like nonzero constants and in $\$ 3$ we deal with solutions which behave asymptotically like $c t$ $(c \neq 0)$. The results of $\S \S 2$ and 3 are almost parallel.

Related results are also contained in Hartman [5, Chapter 11, §9], Hartman and Wintner [6], Trench [11, 12], and Wintner [14]. In the papers of Trench, equations of higher order are considered.

Before stating and proving the main results we give a theorem which is concerned with a nonoscillatory solution of (1.1).

THEOREM 1.1. Suppose $x(t)$ is a nonoscillatory solution of $(1.1)$ on $[T, \infty)$. Then the integral equation

$$
\frac{x^{\prime}(t)}{f(x(t))}=\alpha+A(t)+\int_{t}^{\infty} \frac{f^{\prime}(x(s))\left[x^{\prime}(s)\right]^{2}}{[f(x(s))]^{2}} d s
$$

is satisfied for $t \geqslant T$, where $\alpha$ is a nonnegative constant. Moreover, if the condition

$$
\lim _{x \rightarrow \pm \infty} f(x)= \pm \infty
$$

is satisfied, then $\alpha=0$.

This theorem has been proved by Kwong and Wong [9] and is very useful in the following sections.

2. Bounded asymptotically linear solutions. In this section we obtain necessary and/or sufficient conditions for (1.1) to have solutions which behave asymptotically like nonzero constants.

THEOREM 2.1. Suppose

$$
\begin{aligned}
& \int^{\infty}|A(s)| d s<\infty, \\
& \int^{\infty} s A^{2}(s) d s<\infty .
\end{aligned}
$$

Then, for any $c \neq 0,(1.1)$ has a solution $x(t)$ such that

$$
\begin{gathered}
x(t)=c+O\left(\int_{t}^{\infty}\{|A(s)|+B(s)\} d s\right), \\
x^{\prime}(t)=O(|A(t)|+B(t))
\end{gathered}
$$

as $t \rightarrow \infty$, where $B(t)=\int_{t}^{\infty} A^{2}(s) d s$.

Proof. Condition (2.2) implies $B(t)$ is nonincreasing and integrable on $[0, \infty)$. We may assume $c>0$. Put

$$
m=\max \{f(x): x \in[c / 2,3 c / 2]\}, \quad m^{\prime}=\max \left\{\left|f^{\prime}(x)\right|: x \in[c / 2,3 c / 2]\right\}
$$

and choose constants $b, T>0$ so that

$$
m m^{\prime}+b m^{\prime} \int_{T}^{\infty}|A(s)| d s \leqslant b,
$$




$$
m \int_{T}^{\infty}|A(s)| d s+b \int_{T}^{\infty} B(s) d s \leqslant \frac{c}{2} .
$$

Let $F$ be the Fréchet space of all continuously differentiable functions on $[T, \infty)$ with the family of seminorms $\left\{\|\cdot\|_{l}: l=1,2, \ldots\right\}$ defined by

$$
\|x\|_{l}=\sup \left\{|x(t)|+\left|x^{\prime}(t)\right|: T \leqslant t \leqslant T+l\right\} .
$$

We have the convergence $x_{k} \rightarrow x(k \rightarrow \infty)$ in the topology of $F$ if and only if $x_{k}(t) \rightarrow x(t)(k \rightarrow \infty)$ and $x_{k}^{\prime}(t) \rightarrow x^{\prime}(t)(k \rightarrow \infty)$ uniformly on every compact subinterval of $[T, \infty)$. Let $X$ denote the set of functions $x \in F$ such that $|x(t)-c| \leqslant$ $c / 2,\left|x^{\prime}(t)\right| \leqslant m|A(t)|+b B(t)$ for $t \geqslant T$. Note that $X$ is a nonempty closed convex subset of $F$. Define the operator $\Phi$ on $X$ by

$$
(\Phi x)(t)=c-\int_{t}^{\infty} A(s) f(x(s)) d s-\int_{t}^{\infty}\left(\int_{s}^{\infty} A(u) f^{\prime}(x(u)) x^{\prime}(u) d u\right) d s
$$

for $t \geqslant T$. We seek a fixed point of $\Phi$ in $X$ with the aid of the Schauder-Tychonoff theorem.

(i) $\Phi$ is well defined on $X$ and maps $X$ into itself. Let $x \in X$. We have for $r \geqslant s \geqslant T$

$$
\begin{aligned}
\left|\int_{s}^{r} A(u) f^{\prime}(x(u)) x^{\prime}(u) d u\right| & \leqslant \int_{s}^{r}|A(u)| m^{\prime}\{m|A(u)|+b B(u)\} d u \\
& \leqslant m m^{\prime} \int_{s}^{r} A^{2}(u) d u+m^{\prime} b B(s) \int_{s}^{r}|A(u)| d u .
\end{aligned}
$$

Therefore, letting $r \rightarrow \infty$ and using (2.5), we see that the integral of $A(u) f^{\prime}(x(u)) x^{\prime}(u)$ over $[s, \infty)$ converges and satisfies

$$
\left|\int_{s}^{\infty} A(u) f^{\prime}(x(u)) x^{\prime}(u) d u\right| \leqslant b B(s)
$$

for $s \geqslant T$. This implies

$$
\begin{aligned}
\left|(\Phi x)^{\prime}(t)\right| & \leqslant|A(t) f(x(t))|+\left|\int_{t}^{\infty} A(u) f^{\prime}(x(u)) x^{\prime}(u) d u\right| \\
& \leqslant m|A(t)|+b B(t)
\end{aligned}
$$

for $t \geqslant T$, and in view of (2.6) we have

$$
\begin{aligned}
|(\Phi x)(t)-c| \leqslant & \int_{T}^{\infty}|A(s) f(x(s))| d s \\
& +\int_{T}^{\infty}\left|\int_{s}^{\infty} A(u) f^{\prime}(x(u)) x^{\prime}(u) d u\right| d s \\
\leqslant & c / 2
\end{aligned}
$$

for $t \geqslant T$. Thus $\Phi$ is well defined on $X$ and maps $X$ into $X$.

(ii) $\Phi$ is continuous on $X$. Let $x, x_{k}(k=1,2, \ldots)$ be functions in $X$ such that $x_{k}(t) \rightarrow x(t), x_{k}^{\prime}(t) \rightarrow x^{\prime}(t)$ as $k \rightarrow \infty$ uniformly on every compact subinterval of $[T, \infty)$. Then we have

$$
\begin{aligned}
\left|\left(\Phi x_{k}\right)^{\prime}(t)-(\Phi x)^{\prime}(t)\right| \leqslant & |A(t)|\left|f\left(x_{k}(t)\right)-f(x(t))\right| \\
& +\int_{t}^{\infty}\left|A(u) \| f^{\prime}\left(x_{k}(u)\right) x_{k}^{\prime}(u)-f^{\prime}(x(u)) x^{\prime}(u)\right| d u
\end{aligned}
$$


for $t \geqslant T$. Observe that $f\left(x_{k}(t)\right) \rightarrow f(x(t))$ as $k \rightarrow \infty$ uniformly on compact subintervals of $[T, \infty)$ and $\left|A(u) \| f^{\prime}\left(x_{k}(u)\right) x_{k}^{\prime}(u)-f^{\prime}(x(u)) x^{\prime}(u)\right|$ is bounded above by the integrable function $2 m^{\prime}|A(u)|\{m|A(u)|+b B(u)\}$ on $[T, \infty)$, and $|A(u)|\left|f^{\prime}\left(x_{k}(u)\right) x_{k}^{\prime}(u)-f^{\prime}(x(u)) x^{\prime}(u)\right|$ tends to 0 as $k \rightarrow \infty$. Applying the Lebesgue dominated convergence theorem, we find that $\left(\Phi x_{k}\right)^{\prime}(t) \rightarrow(\Phi x)^{\prime}(t)$ as $k \rightarrow \infty$ uniformly on every compact subinterval of $[T, \infty)$. Moreover, since

$$
\left|\left(\Phi x_{k}\right)(t)-(\Phi x)(t)\right| \leqslant \int_{t}^{\infty}\left|\left(\Phi x_{k}\right)^{\prime}(s)-(\Phi x)^{\prime}(s)\right| d s
$$

and $\left|\left(\Phi x_{k}\right)^{\prime}(s)-(\Phi x)^{\prime}(s)\right|$ is bounded above by the integrable function $2\{m|A(s)|+b B(s)\}$ on $[T, \infty)$, using the Lebesgue dominated convergence theorem again, we conclude that $\left(\Phi x_{k}\right)(t) \rightarrow(\Phi x)(t)$ as $k \rightarrow \infty$ uniformly on every finite subinterval of $[T, \infty)$. Thus $\Phi$ is continuous on $X$.

(iii) $\overline{\Phi X}$ is compact. This can be proved with the aid of Ascoli's theorem. Since the proof is standard, the details are omitted.

From the preceding considerations we are able to apply the Schauder-Tychonoff fixed point theorem to the operator $\Phi$ defined on $X$. Let $x \in X$ be a fixed point of $\Phi$. It is immediately verified that $x=x(t)$ is a solution of (1.1) for $t>T$ and has properties (2.3) and (2.4). This completes the proof of Theorem 2.1.

REMARK. In Theorem 2.1 the assumption of $f^{\prime}(x) \geqslant 0(x \neq 0)$ is unnecessary. In fact, this sign condition of $f^{\prime}$ is nowhere used in the proof.

It is known that if (2.1) and (2.2) are satisfied, then (1.1) has a nonoscillatory solution (Butler [2]). Theorem 2.1 asserts more strongly that under the same conditions (1.1) has a nonoscillatory solution with the asymptotic behavior (2.3) and (2.4). As an example consider the equation

$$
x^{\prime \prime}+\left(k t^{\lambda} \sin t\right)|x|^{\gamma} \operatorname{sgn} x=0, \quad t>0
$$

where $k, \lambda$ and $\gamma>0$ are constants. Applying Theorem 2.1 to the case $f(x)=$ $|x|^{\gamma} \operatorname{sgn} x$

$$
|A(t)|=\left|\int_{t}^{\infty} k s^{\lambda} \sin s d s\right| \leqslant 2|k| t^{\lambda} \quad(\lambda<0),
$$

we find that if $\lambda<-1$, then, for any $c \neq 0$, (2.8) has a solution $x(t)$ such that $x(t)=c+O\left(t^{\lambda+1}\right), x^{\prime}(t)=O\left(t^{\lambda}\right)$ as $t \rightarrow \infty$. Note that (2.8) has a nonoscillatory solution if and only if

$$
\begin{aligned}
& \lambda<-1 \text { for } \gamma>1 \quad \text { (Butler [2-4]); } \\
& \lambda<-1, k \text { arbitrary }\} \quad \text { for } \gamma=1 \quad \text { (Willett [13]), } \\
& \lambda=-1,|k| \leqslant 1 / \sqrt{2}\} \quad \text { for } \gamma=1 \quad \text { (Willett [13], Wong [16]); } \\
& \lambda<-\gamma \text { for } 0<\gamma<1 \quad \text { (Kura [7], Onose [10], Kwong and Wong [8]). }
\end{aligned}
$$

The superlinear case $(\gamma>1)$ shows that our result is "sharp" in the sense that (2.8) never has a nonoscillatory solution unless $\lambda<-1$.

We have the following results as corollaries of Theorem 2.1. 
COROLlary 2.2. Suppose (2.1) and (2.2) are satisfied. Then, for any $c \neq 0,(1.1)$ has a nonoscillatory solution $x(t)$ such that

$$
x(t)=c+o(1) \text { as } t \rightarrow \infty .
$$

COROllary 2.3. Suppose (2.1) and

$$
t A(t) \rightarrow 0 \quad(t \rightarrow \infty)
$$

are satisfied. Then, for any $c \neq 0,(1.1)$ has a nonoscillatory solution $x(t)$ such that

$$
x(t)=c+o(1), \quad x^{\prime}(t)=o\left(t^{-1}\right) \quad \text { as } t \rightarrow \infty .
$$

Corollary 2.2 is a direct consequence of Theorem 2.1. For the proof of Corollary 2.3 we have only to note that (2.1) and (2.10) imply (2.2), and

$$
t \int_{t}^{\infty} A^{2}(s) d s \leqslant \int_{t}^{\infty} s A^{2}(s) d s \rightarrow 0 \quad(t \rightarrow \infty) .
$$

REMARK. In Corollary 2.2, if (1.1) is linear, then the absolute integrability of $A(t)$ (i.e. condition (2.1)) can be replaced by the conditional integrability of $A(t)$ (Hartman and Wintner [6] or Hartman [5, Corollary 9.3, p. 382]).

In what follows it is shown that the converse of Corollaries 2.2 and 2.3 in some sense can be obtained when $A(t)$ does not change sign.

Theorem 2.4. Suppose $A(t) \geqslant 0$ for all large $t$ and $f^{\prime}(x)>0$ for $x \neq 0$. Then the following statements are equivalent:

(i) for any $c \neq 0$, there exists a solution $x(t)$ of (1.1) satisfying (2.9);

(ii) for some $c \neq 0$, there exists a solution $x(t)$ of (1.1) satisfying (2.9);

(iii) the integral conditions (2.1) and (2.2) are satisfied.

Proof. (i) implies (ii) trivially, and (iii) implies (i) by Corollary 2.2. We claim (ii) implies (iii). Let $x(t)$ be a solution of (1.1) for which (2.9) holds for some $c \neq 0$. We may assume $c>0$. There is a number $T$ such that $c / 2 \leqslant x(t) \leqslant 2 c$ for $t \geqslant T$. By Theorem 1.1, (1.5) with $\alpha$ nonnegative is satisfied for $t \geqslant T$. It is easy to verify that, under the condition $A(t) \geqslant 0$,

$$
\frac{x^{\prime}(t)}{f(x(t))} \geqslant A(t)+\int_{t}^{\infty} m^{\prime} A^{2}(s) d s \geqslant 0
$$

for $t \geqslant T$, where $m^{\prime}=\min \left\{f^{\prime}(x): c / 2 \leqslant x \leqslant 2 c\right\}>0$. An integration over $[T, t]$ gives

$$
\int_{x(T)}^{x(t)} \frac{d y}{f(y)} \geqslant \int_{T}^{t}\left(A(s)+m^{\prime} \int_{s}^{\infty} A^{2}(u) d u\right) d s
$$

for $t \geqslant T$. Since the left-hand side of the above remains bounded as $t \rightarrow \infty$, we conclude that (2.1) and (2.2) are satisfied. The proof of Theorem 2.4 is complete.

THEOREM 2.5. Suppose either $A(t) \geqslant 0$ or $A(t) \leqslant 0$ for all large $t$. Then the following statements are equivalent:

(i) for any $c \neq 0$, there exists a solution $x(t)$ of (1.1) satisfying (2.11);

(ii) for some $c \neq 0$, there exists a solution $x(t)$ of (1.1) satisfying (2.11);

(iii) the conditions (2.1) and (2.10) are satisfied. 
Proof. As (i) implies (ii) trivially and (iii) implies (i) by Corollary 2.3 , it suffices to show that (ii) implies (iii). Suppose $x(t)$ is a solution of (1.1) such that (2.11) holds for some $c \neq 0$. From Theorem 1.1, (1.5) is satisfied for all large $t$. Since $\alpha=$ $\lim _{t \rightarrow x} x^{\prime}(t) / f(x(t))=0$, we have

$$
\frac{x^{\prime}(t)}{f(x(t))}=A(t)+\int_{t}^{\infty} \frac{f^{\prime}(x(s))\left[x^{\prime}(s)\right]^{2}}{[f(x(s))]^{2}} d s
$$

or, equivalently,

$$
t A(t)=\frac{t x^{\prime}(t)}{f(x(t))}-t \int_{t}^{\infty} \frac{f^{\prime}(x(s))\left[x^{\prime}(s)\right]^{2}}{[f(x(s))]^{2}} d s
$$

for all large $t$. The first term of the right-hand side of (2.12) tends to 0 as $t \rightarrow \infty$ since $x(t)$ satisfies (2.11). Using l'Hospital's rule and (2.11), we find that the second term of the right-hand side of (2.12) tends to 0 as $t \rightarrow \infty$ :

$$
\begin{aligned}
\left(\frac{d}{d t} \int_{t}^{\infty} \frac{f^{\prime}(x(s))\left[x^{\prime}(s)\right]^{2}}{[f(x(s))]^{2}} d s\right)\left(\frac{d}{d t} t^{-1}\right)^{-1} & =\frac{f^{\prime}(x(t))}{[f(x(t))]^{2}}\left[t x^{\prime}(t)\right]^{2} \\
& \rightarrow 0 \text { as } t \rightarrow \infty
\end{aligned}
$$

Therefore we have (2.10).

In order to prove that (2.1) holds, we may use the technique in Hartman and Wintner [6]. According to (1.1), we get

$$
\begin{aligned}
\left(x(t)-t x^{\prime}(t)\right)^{\prime} & =t a(t) f(x(t)) \\
& =-(t A(t) f(x(t)))^{\prime}+A(t) f(x(t))+t A(t) f^{\prime}(x(t)) x^{\prime}(t) .
\end{aligned}
$$

An integration of the above gives

$$
\begin{aligned}
x(t)-t x^{\prime}(t)= & \text { const }-t A(t) f(x(t)) \\
& +\int_{T}^{t} A(s)\left[f(x(s))+s f^{\prime}(x(s)) x^{\prime}(s)\right] d s,
\end{aligned}
$$

where const $=x(T)-T x^{\prime}(T)+T A(T) f(x(T))$. Since $t A(t) \rightarrow 0(t \rightarrow \infty)$ and $x(t)$ satisfies (2.11), we have $x(t)-t x^{\prime}(t) \rightarrow c, \quad t A(t) f(x(t)) \rightarrow 0$, and $f(x(t))+$ $t x^{\prime}(t) f^{\prime}(x(t)) \rightarrow f(c) \neq 0$ as $t \rightarrow \infty$. In view of these facts combined with (2.13) and the sign condition of $A(t)$ we can easily verify that $(2.1)$ holds. This completes the proof of Theorem 2.5 .

From Theorems 2.4 and 2.5 we see that, even for solutions which have the same limits as $t \rightarrow \infty$, there is an essential difference between restricting and not restricting the asymptotic behavior of the derivatives of the solutions.

Very recently Trench [12] proved that, among other results, if $a(t)$ satisfies

$$
\lim _{t \rightarrow \infty} \int^{t} s a(s) d s \text { exists and is finite, and } \int^{\infty}|A(s)| d s<\infty,
$$

then for any $c \neq 0,(1.2)$ has a solution $x(t)$ satisfying (2.11). But it is not difficult to show that conditions (2.1) and (2.10) together are equivalent to (2.14). Moreover, it can be shown that if either $A(t) \geqslant 0$ or $A(t) \leqslant 0$ for all large $t$, then conditions (2.1) 
and (2.10) together are equivalent to the condition that

$$
\lim _{t \rightarrow \infty} \int^{t} s a(s) d s \text { exists and is finite. }
$$

(In other words the absolute integrability of $A(t)$ in (2.14) is superfluous when $A(t)$ does not change sign.) Consequently, we see that condition (iii) of Theorem 2.5 may be replaced by $(2.15)$.

3. Unbounded asymptotically linear solutions. The purpose of this section is to obtain necessary and/or sufficient conditions for (1.1) to have solutions which behave asymptotically like $c t(c \neq 0)$. In the preceding section, no growth condition on $f$ was required in proving the existence of a solution asymptotic to a nonzero constant as $t \rightarrow \infty$. The situation becomes different now and we shall impose one of the following growth conditions on $f$ :

(3.1a) $f^{\prime}(x)$ is nondecreasing for $x>0$ and nonincreasing for $x<0$;

(3.1b) $\quad f^{\prime}(x)$ is nonincreasing for $x>0$ and nondecreasing for $x<0$.

TheOrem 3.1. Suppose either (3.1a) or (3.1b) is satisfied. Also suppose

$$
\begin{gathered}
\frac{1}{t} \int^{t}|f(k s)||A(s)| d s \rightarrow 0 \quad(t \rightarrow \infty) \quad \text { for every } k \neq 0, \\
\int^{\infty} f^{\prime}(k s)|A(s)| d s<\infty \quad \text { for every } k \neq 0, \\
\int^{\infty}\left|f\left(k_{1} s\right)\right| f^{\prime}\left(k_{2} s\right) A^{2}(s) d s<\infty \quad \text { for every } k_{1}, k_{2} \neq 0 .
\end{gathered}
$$

Then, for any $c \neq 0,(1.1)$ has a solution $x(t)$ such that

$$
\begin{gathered}
x(t)=c t+O\left(\int^{t}\left\{|f(\bar{c} s)||A(s)|+B_{c}(s)\right\} d s\right), \\
x^{\prime}(t)=c+O\left(|f(\bar{c} t)||A(t)|+B_{c}(t)\right)
\end{gathered}
$$

as $t \rightarrow \infty$, where

$$
B_{c}(t)=\sup _{r \geqslant t} \max \left\{\int_{r}^{\infty} f^{\prime}(\tilde{c} s)|A(s)| d s, \int_{r}^{\infty}|f(\bar{c} s)| f^{\prime}(\tilde{c} s) A^{2}(s) d s\right\},
$$

and $\bar{c}=3 c / 2, \tilde{c}=3 c / 2$ (if (3.1a) holds), $\tilde{c}=c / 2$ (if (3.1b) holds).

Proof. We outline the proof. The details are left to the reader. Let $c$ be a given nonzero number. Without loss of generality we may assume $c>0$. By (3.2), (3.3) and the fact that $B_{c}(t) \rightarrow 0(t \rightarrow \infty)$, there is a sufficiently large $T$ such that the following three conditions are satisfied for $t \geqslant T$ :

$$
\begin{aligned}
& \int_{T}^{t} f(\bar{c} s)|A(s)| d s \leqslant \frac{c}{4} t, \\
& (c+2) \int_{t}^{\infty} f^{\prime}(\tilde{c} u)|A(u)| d u \leqslant 1, \\
& (c+2) \int_{T}^{t} B_{c}(s) d s \leqslant \frac{c}{4} t .
\end{aligned}
$$


Let $F$ be the Fréchet space of all continuously differentiable functions on $[T, \infty)$ with the topology as defined in the proof of Theorem 2.1, and let $X$ denote the set of functions $x \in F$ such that $|x(t)-c t| \leqslant(c / 2) t, \quad\left|x^{\prime}(t)\right| \leqslant c+f(\bar{c} t)|A(t)|+$ $(c+2) B_{c}(t)$ for $t \geqslant T$. Clearly, $X$ is a nonempty closed convex subset of $F$. Then consider a mapping $\Phi: X \rightarrow F$ defined by

$$
(\Phi x)(t)=c t+\int_{T}^{t} A(s) f(x(s)) d s+\int_{T}^{t}\left(\int_{s}^{\infty} A(u) f^{\prime}(x(u)) x^{\prime}(u) d u\right) d s
$$

for $t \geqslant T$. As in the proof of Theorem 2.1 it can be shown that (i) $\Phi$ is well defined on $X$ and maps $X$ into $X$, (ii) $\Phi$ is continuous on $X$, and (iii) $\overline{\Phi X}$ is compact. By the Schauder-Tychonoff fixed point theorem the operator $\Phi$ has a fixed point $x \in X$. This fixed point $x=x(t)$ provides a solution of (1.1) satisfying (3.5) and (3.6).

As an example we again consider (2.8). Applying Theorem 3.1 to the case $f(x)=|x|^{\gamma} \operatorname{sgn} x,|A(t)| \leqslant 2|k| t^{\lambda}(\lambda<0)$, we see that if $\lambda<-\gamma$, then (2.8) has a solution $x(t)$ such that $x(t)=c t+O\left(t^{\delta}\right)$, where $\delta=\max \{\lambda+\gamma+1,0\}$ if $\lambda+\gamma+$ $1 \neq 0$ and $x(t)=c t+O(\log t)$ if $\lambda+\gamma+1=0$. Theorem 3.1 is also "sharp" in the sense that $(2.8)$ in the sublinear case $(0<\gamma<1)$ never has a nonoscillatory solution unless $\lambda<-\gamma$.

As corollaries of Theorem 3.1 we have the following results.

COROllary 3.2. Suppose either (3.1a) or (3.1b) is satisfied. Also suppose (3.2)-(3.4) are satisfied. Then, for any $c \neq 0,(1.1)$ has a solution $x(t)$ such that

$$
x(t)=t[c+o(1)] \text { as } t \rightarrow \infty .
$$

Corollary 3.3. Suppose either (3.1a) or (3.1b) is satisfied. Also suppose

$$
\begin{gathered}
f(k t) A(t) \rightarrow 0 \quad(t \rightarrow \infty) \quad \text { for every } k \neq 0, \\
\int^{\infty} f^{\prime}(k s)|A(s)| d s<\infty \quad \text { for every } k \neq 0 .
\end{gathered}
$$

Then, for any $c \neq 0,(1.1)$ has a solution $x(t)$ such that

$$
x(t)=t[c+o(1)], \quad x^{\prime}(t)=c+o(1) \quad \text { as } t \rightarrow \infty .
$$

We can prove the converse of Corollaries 3.2 and 3.3 when $A(t)$ does not change sign.

TheOREM 3.4. Suppose $A(t) \geqslant 0$ for all large $t$. Moreover, in addition to (3.1a) or (3.1b), suppose $f^{\prime}(x)>0$ for $x \neq 0$, and

$$
\limsup _{t \rightarrow \infty} \frac{1}{t^{2}} \int^{t}\left|f\left(k_{1} s\right)\right|\left[f^{\prime}\left(k_{2} s\right)\right]^{-1} d s<\infty \text { for every } k_{1}, k_{2} \neq 0 .
$$

Then in order for (1.1) to have a solution $x(t)$ satisfying (3.7) for some $c \neq 0$ it is necessary that:

$$
\begin{gathered}
\frac{1}{t} \int^{t}|f(k s)| A(s) d s \rightarrow 0 \quad(t \rightarrow \infty) \quad \text { for some } k \neq 0 \\
\int^{\infty} f^{\prime}(k s) A(s) d s<\infty \quad \text { for some } k \neq 0
\end{gathered}
$$




$$
\int^{\infty}\left|f\left(k_{1} s\right)\right| f^{\prime}\left(k_{2} s\right) A^{2}(s) d s<\infty \text { for some } k_{1}, k_{2} \neq 0 .
$$

Proof. Let $x(t)$ be a solution of (1.1) which satisfies (3.7). We may assume $c>0$. There is a number $T$ such that $(c / 2) t \leqslant x(t) \leqslant 2 c t$ for $t \geqslant T$. From Theorem 1.1 it follows that

$$
\begin{aligned}
x^{\prime}(t)= & \alpha f(x(t))+A(t) f(x(t)) \\
& +f(x(t)) \int_{t}^{\infty} \frac{f^{\prime}(x(s))\left[x^{\prime}(s)\right]^{2}}{[f(x(s))]^{2}} d s
\end{aligned}
$$

for $t \geqslant T$, where $\alpha$ is a nonnegative constant. On the other hand, an integration by parts of (1.1) gives

$$
x^{\prime}(t)=\mathrm{const}+A(t) f(x(t))-\int_{T}^{t} A(s) f^{\prime}(x(s)) x^{\prime}(s) d s,
$$

where const $=x^{\prime}(T)-A(T) f(x(T))$. Combining (3.15) with (3.16), we have

$$
\begin{gathered}
\alpha f(x(t))+f(x(t)) \int_{t}^{\infty} \frac{f^{\prime}(x(s))\left[x^{\prime}(s)\right]^{2}}{[f(x(s))]^{2}} d s \\
=\text { const }-\int_{T}^{t} A(s) f^{\prime}(x(s)) x^{\prime}(s) d s .
\end{gathered}
$$

Since $x^{\prime}(t) \geqslant 0$ by (3.15), (3.17) implies

$$
\int_{T}^{\infty} A(s) f^{\prime}(x(s)) x^{\prime}(s) d s<\infty .
$$

Using the inequality $x^{\prime}(t) \geqslant A(t) f(x(t))(t \geqslant T)$, which is derived from (3.15), we conclude that

$$
\int_{T}^{\infty} f(x(s)) f^{\prime}(x(s)) A^{2}(s) d s<\infty,
$$

which, in view of (3.1), implies (3.14). By (3.18) we find that the right-hand side of (3.17) has a finite limit as $t \rightarrow \infty$. Let $\beta$ denote the limit:

$$
\beta=\lim _{t \rightarrow \infty}\left\{\alpha f(x(t))+f(x(t)) \int_{t}^{\infty} \frac{f^{\prime}(x(s))\left[x^{\prime}(s)\right]^{2}}{[f(x(s))]^{2}} d s\right\} .
$$

Integrating (3.15) over $[\tau, t](t \geqslant \tau \geqslant T)$ and dividing by $t$, we get

$$
\begin{aligned}
\frac{x(t)-x(\tau)}{t}= & \frac{1}{t} \int_{\tau}^{t} A(s) f(x(s)) d s+\frac{\alpha}{t} \int_{\tau}^{t} f(x(s)) d s \\
& +\frac{1}{t} \int_{\tau}^{t} f(x(s))\left(\int_{s}^{\infty} \frac{f^{\prime}(x(u))\left[x^{\prime}(u)\right]^{2}}{[f(x(u))]^{2}} d u\right) d s .
\end{aligned}
$$

Since $x(t)$ satisfies (3.7), from (3.19) and (3.20) it follows that

$$
\lim _{t \rightarrow \infty} \frac{1}{t} \int_{\tau}^{t} A(s) f(x(s)) d s=c-\beta .
$$


It is easy to see that

$$
0 \leqslant \frac{1}{t} \int_{\tau}^{t} A(s) f(x(s)) d s \leqslant \frac{1}{t}\left(\int_{\tau}^{t} \frac{f(2 c s)}{f^{\prime}\left(c^{\prime} s\right)} d s\right)^{1 / 2}\left(\int_{\tau}^{t} f(x(s)) f^{\prime}(x(s)) A^{2}(s) d s\right)^{1 / 2}
$$

for $t \geqslant \tau$, where $c^{\prime}=c / 2$ if (3.1a) holds and $c^{\prime}=2 c$ if (3.1b) holds. Note that (3.11) implies there exists a positive constant $M$ independent of $\tau$ such that

$$
\frac{1}{t^{2}} \int_{\tau}^{t} \frac{f(2 c s)}{f^{\prime}\left(c^{\prime} s\right)} d s \leqslant M^{2}
$$

for $t \geqslant \tau$. Taking the limit as $t \rightarrow \infty$ in (3.22), we have, from (3.21),

$$
0 \leqslant c-\beta \leqslant M\left(\int_{\tau}^{\infty} f(x(s)) f^{\prime}(x(s)) A^{2}(s) d s\right)^{1 / 2} .
$$

Since $\tau$ is arbitrary, letting $\tau \rightarrow \infty$ in (3.23), we see that $c=\beta$. Therefore, we have from (3.19) and (3.21) that

$$
\begin{gathered}
\lim _{t \rightarrow \infty}\left\{\alpha f(x(t))+f(x(t)) \int_{t}^{\infty} \frac{f^{\prime}(x(s))\left[x^{\prime}(s)\right]^{2}}{[f(x(s))]^{2}} d s\right\}=c, \\
\lim _{t \rightarrow \infty} \frac{1}{t} \int^{t} A(s) f(x(s)) d s=0 .
\end{gathered}
$$

In view of (3.25) we find that (3.12) is satisfied. By (3.15) and (3.24), we obtain $x^{\prime}(t) \geqslant c / 2$ for all large $t$. Combining this with (3.18), we see that (3.13) is satisfied. This completes the proof of Theorem 3.4.

When applied to the special case of the generalized Emden-Fowler equation (1.2), Corollary 3.2 and Theorem 3.4 yield the following result.

THEOREM 3.5. In (1.2) suppose $A(t) \geqslant 0$ for all large $t$. Then the following statements are equivalent:

(i) for any $c \neq 0$, there exists a solution $x(t)$ of (1.2) satisfying (3.7);

(ii) for some $c \neq 0$, there exists a solution $x(t)$ of (1.2) satisfying (3.7);

(iii) the two integral conditions below are satisfied:

$$
\begin{gathered}
\int^{\infty} s^{\gamma-1} A(s) d s<\infty, \\
\int^{\infty} s^{2 \gamma-1} A^{2}(s) d s<\infty .
\end{gathered}
$$

For the proof we have only to notice that the condition $t^{-1} \int^{t} s^{\gamma} A(s) d s \rightarrow 0$ $(t \rightarrow \infty)$, which corresponds to (3.2), is implied by (3.26).

TheOREM 3.6. Suppose either $A(t) \geqslant 0$ or $A(t) \leqslant 0$ for all large $t$. Also suppose either (3.1a) or (3.1b) is satisfied. Then in order for (1.1) to have a solution $x(t)$ satisfying (3.10) for some $c \neq 0$ it is necessary that:

$$
\begin{gathered}
f(k t) A(t) \rightarrow 0 \quad(t \rightarrow \infty) \quad \text { for some } k \neq 0, \\
\int^{\infty} f^{\prime}(k s)|A(s)| d s<\infty \quad \text { for some } k \neq 0 .
\end{gathered}
$$


Proof. First we prove (3.28). If $f(x)$ is bounded as $x \rightarrow \infty$ or $-\infty$, then (3.28) is trivially satisfied since $A(t) \rightarrow 0(t \rightarrow \infty)$. Thus we may assume $\lim _{x \rightarrow \pm \infty} f(x)=$ $\pm \infty$. Let $x(t)$ be a solution of (1.1) satisfying (3.10). By Theorem 1.1 we have

$$
A(t) f(x(t))=x^{\prime}(t)-f(x(t)) \int_{t}^{\infty} \frac{f^{\prime}(x(s))\left[x^{\prime}(s)\right]^{2}}{[f(x(s))]^{2}} d s
$$

for $t \geqslant T$. Using l'Hospital's rule, we see that the second term of the right-hand side of (3.30) tends to $c$ as $t \rightarrow \infty$ :

$$
\left(\frac{d}{d t} \int_{t}^{\infty} \frac{f^{\prime}(x(s))\left[x^{\prime}(s)\right]^{2}}{[f(x(s))]^{2}} d s\right)\left(\frac{d}{d t}[f(x(t))]^{-1}\right)^{-1}=x^{\prime}(t) \rightarrow c \quad \text { as } t \rightarrow \infty .
$$

It follows from (3.30) and (3.10) that $A(t) f(x(t)) \rightarrow 0$ as $t \rightarrow \infty$, which implies (3.28) is satisfied. Integrating (1.1) by parts, we have (3.16). Since $x^{\prime}(t) \rightarrow c, A(t) f(x(t)) \rightarrow 0$ $(t \rightarrow \infty)$, we see that

$$
\int^{\infty}|A(s)| f^{\prime}(x(s))\left|x^{\prime}(s)\right| d s<\infty,
$$

and from this (3.29) readily follows. The proof is complete.

Combining Corollary 3.3 with Theorem 3.6, we have the following result.

THEOREM 3.7. In (1.2) suppose either $A(t) \geqslant 0$ or $A(t) \leqslant 0$ for all large $t$. Then the following statements are equivalent:

(i) for any $c \neq 0$, there exists a solution $x(t)$ of (1.2) satisfying (3.10);

(ii) for some $c \neq 0$, there exists a solution $x(t)$ of (1.2) satisfying (3.10);

(iii) the two conditions below are satisfied:

$$
\begin{gathered}
t^{\gamma} A(t) \rightarrow 0 \quad(t \rightarrow \infty), \\
\int^{\infty} s^{\gamma-1}|A(s)| d s<\infty .
\end{gathered}
$$

It is easily seen that Corollary 3.3 is a generalization of a result of Kwong and Wong [8, Theorem 2]. Trench [12] showed that, if $a(t)$ satisfies

(3.33) $\lim _{t \rightarrow \infty} \int^{t} s^{\gamma} a(s) d s$ exists and is finite, and $\int^{\infty} s^{\gamma-1}|A(s)| d s<\infty$,

then (1.2) has a solution $x(t)$ satisfying (3.10). It can be verified that conditions (3.31) and (3.32) together are equivalent to (3.33), and that if either $A(t) \geqslant 0$ or $A(t) \leqslant 0$ for all large $t$ then (3.31) and (3.32) together are equivalent to

$$
\lim _{t \rightarrow \infty} \int^{t} s^{\gamma} a(s) d s \quad \text { exists and is finite. }
$$

Thus condition (iii) of Theorem 3.7 may be replaced by (3.34).

\section{REFERENCES}

1. N. P. Bhatia, Some oscillation theorems for second order differential equations, J. Math. Anal. Appl. 15 (1966), 442-446.

2. G. J. Butler, On the oscillatory behaviour of a second order nonlinear differential equation, Ann. Mat. Pura Appl. 105 (1975), 73-92. 
3. Oscillation theorems for a nonlinear analogue of Hill's equation, Quart. J. Math. Oxford Ser. (2) 27 (1976), 159-171

4. Integral averages and the oscillation of second order ordinary differential equations, SIAM J. Math. Anal. 11 (1980), 190-200.

5. P. Hartman, Ordinary differential equations, Wiley, New York, 1964.

6. P. Hartman and A. Wintner, On non-oscillatory linear differential equations, Amer. J. Math. 75 (1953), 717-730.

7. T. Kura, Oscillation theorems for a second order sublinear ordinary differential equation, Proc. Amer. Math. Soc. 84 (1982), 535-538.

8. M. K. Kwong and J. S. W. Wong, On the oscillation and nonoscillation of second order sublinear equations, Proc. Amer. Math. Soc. 85 (1982), 547-551.

9. __ An application of integral inequality to second order nonlinear oscillation, J. Differential Equations 46 (1982), 63-77.

10. H. Onose, On Butler's conjecture for oscillation of an ordinary differential equation, Quart. J. Math. Oxford Ser. (2) 34 (1983), 235-239.

11. W. F. Trench, Asymptotic integration of linear differential equations subject to integral smallness conditions involving ordinary convergence, SIAM J. Math. Anal. 7 (1976), 213-221.

12. A Asymptotic integration of $y^{(n)}+P(t) y^{\gamma}=f(t)$ under mild integral smallness conditions, Funkcial. Ekvac. (to appear).

13. D. Willett, On the oscillatory behavior of the solutions of second order linear differential equations, Ann. Polon. Math. 21 (1969), 175-194.

14. A. Wintner, On almost free linear motions, Amer. J. Math. 71 (1949), 595-602.

15. J. S. W. Wong, On two theorems of Waltman, SIAM J. Appl. Math. 14 (1966), 724-728.

16. , Oscillation and nonoscillation of solutions of second order linear differential equations with integrable coefficients, Trans. Amer. Math. Soc. 144 (1969), 197-215.

Department of Mathematics, Faculty of Science, Hiroshima University, Hiroshima 730 , JAPAN

Current address: Department of Mathematics, Faculty of Education, Tokushima University, Tokushima 770, Japan 